\title{
EL TELETRABAJO: UNA ESTRATEGIA DE MOTIVACIÓN
}

\author{
ADRIANA OVIEDO VEGA \\ Universidad Estatal a Distancia, Costa Rica \\ aoviedo@uned.ac.cr \\ NANCY VÁSQUEZ FLORES \\ Universidad Estatal a Distancia, Costa Rica \\ nvasquez@uned.ac.cr
}

\section{RESUMEN}

El teletrabajo es una modalidad laboral que actualmente se utiliza más en las organizaciones, debido a que contribuye, en una buena parte, a satisfacer las necesidades de los trabajadores. A la vez, existen diversas teorías de motivación que explican las necesidades que todo trabajador tiene y desea satisfacer en todo momento. Es por eso, que bajo la hipótesis de que el teletrabajo le permite, a quien lo ejerce, una mayor calidad de vida, un mejor manejo de su tiempo, una economía en sus gastos de traslados, entre otros, se concluye por medio de esta investigación, que el teletrabajo puede considerarse una estrategia de motivación.

PALABRAS CLAVE: TELETRABAJO, TEORÍAS DE MOTIVACIÓN, CALIDAD DE VIDA, NECESIDADES, PRODUCTIVIDAD.

\section{ABSTRACT}

Telework is a work arrangement that is being increasingly used by organizations to contribute, in good part, to the satisfaction of workers' needs. On the other hand, several Theories of Motivation explain the needs that every worker has and wants to satisfy at all the time. That is why, supposing that teleworking allows employees a better quality of life, a better managing of time, and savings in transportation expenses amongst others things, this essay concludes that teleworking can be considered a motivational strategy.
KEYWORDS: TELEWORK, THEORIES OF MOTIVATION, QUALITY OF LIFE, NEEDS, PRODUCTIVITY.

\section{INTRODUCCIÓN}

Las empresas e instituciones actuales, ante las presiones que ejerce la sociedad del conocimiento, se ven en la obligación de maximizar el uso de las diversas tecnologías de información y comunicación que existen, implementando nuevas modalidades laborales procurando que su organización laboral traiga ventajas tanto al empleador como al trabajador. Todo esto en miras de una modernización de su gestión y una comprensión de que su recurso humano es un capital intangible que le otorga un gran valor a su institución.

Las organizaciones se van renovando por medio de los cambios del entorno y eso hace que, internamente, se generen cambios en su cultura organizacional, clima laboral y demás factores como son: el tema financiero, tecnológico, políticas de personal, entre otros. Cada vez es más difícil contar con funcionarios que se identifiquen con la institución, que sean leales y productivos. Si los cambios que enfrentan las empresas no se manejan de forma oportuna, el recurso humano será una limitante para lograr los objetivos y metas de 
la organización. Para ello, se deben presentar nuevas estrategias a nivel laboral con el fin de lograr la motivación del funcionario y precisamente por medio del teletrabajo se obtendrán esos beneficios que tanto el recurso humano espera para mejorar su calidad de vida.

Las empresas, en la actualidad, anteponen su capacidad de producción y subestiman la importancia y el rol que cumple el recurso humano en ellas. No se analizan las limitaciones que esto le generará a su administración en cuanto a altos índices de rotación, despidos, desmotivación. Esto se resume en altos costos de operación.

Por medio de esta investigación se expondrán las ventajas que les genera a las organizaciones enfocarse en su personal, ofrecer buenas compensaciones y ajustarse a nuevas modalidades como es el teletrabajo.

A partir de esto, se ha planteado como objetivo general: demostrar que el teletrabajo es una estrategia de motivación.

Para alcanzarlo, se considera importante también:

a) Explicar qué es una teoría de motivación y cuáles son las más reconocidas.

b) Definir qué es el teletrabajo y cuáles son las principales ventajas y beneficios que les brinda a las empresas, a la sociedad y al trabajador.

c) Realizar un análisis comparativo entre los beneficios que aporta el teletrabajo y las necesidades que requiere una persona en su vida, según las teorías de motivación.

d) Analizar el aumento de productividad en las empresas, a causa de la implementación del teletrabajo.

\section{METODOLOGÍA}

La metodología utilizada para esta investigación fue cualitativa-teórica en donde la unidad de análisis es el teletrabajo como una estrategia de motivación. A su vez, se desarrolla un artículo documental teórico el cual hace alusión a una investigación descriptiva con el fin de conocer las situaciones, operaciones y actividades mediante la descripción exacta de los procesos y personas, haciendo referencia a estudios de casos de empresas públicas y privadas.

Se deduce en esta investigación que el fin principal es demostrar que el teletrabajo es una estrategia de motivación que pueden aplicar las empresas. A partir de ello se registra, analiza e interpreta la información para presentar de manera descriptiva los respectivos resultados.

En el presente estudio se procede al empleo de tres formas para recolectar la información:

1. Revisión de bibliografía especializada inherente al estudio.

2. Entrevista a Jack Nilles, a quien se le considera mundialmente como el padre del teletrabajo.

3. Análisis de casos de organizaciones que han implementado el teletrabajo, que demuestren si hubo o no aumento de productividad y si las principales necesidades o motivaciones del personal quedaron solventadas.

\section{DESARROLLO}

\section{Un recurso humano motivado}

Al hablar del recurso humano se puede afirmar que es el activo más importante de una organización. Y es por ello que se debe procurar que el recurso humano se sienta motivado.

Según Cofer y Appley (1990) lo que motiva la conducta del ser humano puede ser: "un deter- 
minante ambiental que precipitó la conducta en cuestión, la urgencia, sentimiento, emoción, instinto o carencia, deseo, propósito, demanda, interés, aspiración, plan o necesidad que suscitó la acción y el incentivo meta u objeto de valor que atrajo al organismo" (p. 17).

Asimismo, Villalta (2000) define la motivación como "la realización de aquellas cosas que satisfacen los impulsos y deseos individuales y que lleva a los empleados a actuar de la forma deseada" (p. 94).

En estos tiempos, tanto las instituciones como el personal de las organizaciones, se encuentran inmersos en un constante cambio; no obstante, hay una serie de necesidades básicas que requiere un trabajador, tal y como se puede apreciar en la tabla 1.

Conjuntamente a estas necesidades cada organización debe contar con una política sana sobre su personal que se base en las buenas relaciones y justa compensación para los funcionarios.

Un programa de relaciones con empleados puede no conseguir buena voluntad y entendimiento dentro de una empresa que paga poco, en la cual se trabaja mucho, y hace caso omiso al bienestar de sus funcionarios. Para ello es importante considerar algunas teorías de la motivación que se adecuan a los tiempos de hoy.

El trabajador se desempeña de mejor manera si está motivado y con ello será más productivo. Algunos factores que se le pueden ofrecer al funcionario para que se encuentre motivado son: horarios flexibles, ahorro económico y tiempo libre (si bien es cierto este último no genera inversión directa para la institución, al ofrecerlo logrará que el personal sea más productivo, y generará mayor identidad y empatía con la organización). Casualmente, se podrían ofrecer

\section{NECESIDADES BÁSICAS DE UN TRABAJADOR}

\begin{tabular}{|l|l|}
\hline Estabilidad laboral & $\begin{array}{l}\text { La organización puede ofrecerla por medio de la contratación formal. Se le puede } \\
\text { asignar el liderazgo en un proyecto para que se sienta parte fundamental para el } \\
\text { desarrollo de un trabajo. Asimismo, se debe demostrar que es una empresa seria, } \\
\text { responsable y estable. }\end{array}$ \\
\hline $\begin{array}{l}\text { Crecimiento } \\
\text { profesional }\end{array}$ & $\begin{array}{l}\text { Todo trabajador requiere la posibilidad de perfeccionar sus conocimientos por me- } \\
\text { dio de capacitaciones, ya sea para su puesto actual o puestos futuros. Y a la vez, } \\
\text { tener la posibilidad de asumir nuevos puestos y con ello sentir un crecimiento } \\
\text { profesional. }\end{array}$ \\
\hline Estima & $\begin{array}{l}\text { El funcionario requiere una valoración por su trabajo tanto por parte del jefe, como } \\
\text { de los compañeros y de la organización en la cual labora; además de sentirse } \\
\text { aceptado por ellos. }\end{array}$ \\
\hline Toma de decisión & $\begin{array}{l}\text { Las organizaciones deben ofrecer a sus trabajadores cierta independencia y la po- } \\
\text { testad de que ellos decidan qué es lo más conveniente en su puesto de trabajo. Esto } \\
\text { les dará confianza y reafirmará su estabilidad laboral y el nivel de aceptación que } \\
\text { tienen hacia ese trabajador. }\end{array}$ \\
\hline
\end{tabular}

Fuente: Elaboración propia. 
estos beneficios a sus trabajadores si se implementa la modalidad de teletrabajo.

\section{Teorías de motivación que se relacionan con el teletrabajo}

Superada la revolución industrial, en donde las máquinas tuvieron su protagonismo, hoy inmersos en la sociedad del conocimiento se desarroIla una apertura por el hombre y su entorno social. Es así como distintos pensadores, preocupados por los aspectos psicológicos y sociológicos, dan origen a las teorías de motivación.

De acuerdo con lo anterior, la motivación es esencial para el desempeño individual de un trabajador y el comportamiento organizacional de una empresa o institución, lo que trae por consecuencia una mayor satisfacción y productividad para ambas partes.

Existen diversas teorías que explican el fenómeno de la motivación en el trabajo. Unas podrían ser complementarias con otras, o bien, unas relacionan ciertas variables que coinciden con otras teorías.

A continuación se citan cinco teorías que se aproximan a lo que podría pretender el teletrabajo en una organización y en los individuos.

\section{Jerarquía de Necesidades (Abraham Maslow)}

Maslow (1934) clasifica las motivaciones humanas, psicológicas y físicas según la incidencia en la conducta. Según esta teoría, las necesidades del ser humano se pueden agrupar en cinco niveles:

- Necesidades fisiológicas: necesidades mínimas para que una persona pueda subsistir, como la necesidad de alimento, vestido, vivienda y descanso, entre otros.
- Necesidades de seguridad: la necesidad de la persona de sentirse protegida de amenazas y peligros físicos, económicos o psicológicos.

- Necesidades de afecto o pertenencia: necesidad de pertenecer a cierto grupo social o de amigos.

- Necesidad de estima: deseo de la persona de sentirse respetada por los demás y también el de respetarse a sí misma.

- Necesidad de autorrealización: lleva a la persona a utilizar todas sus potencialidades y habilidades, logrando ser creativa y maximizando todas sus capacidades.

Según Maslow, todas estas necesidades actúan en forma de jerarquía, satisfaciéndose en forma ascendente; es decir, se deben satisfacer primero las necesidades fisiológicas y por último las de autorrealización (Villalta, 2000; Boere, 2003).

\section{Teoría del comportamiento, " $X$ " $y$ " $Y$ " (Douglas McGregor)}

En esta teoría se comparan dos estilos de administrar, uno de manera tradicional y excesiva (Teoría X) y el otro basado en las concepciones del comportamiento humano (Teoría Y).

McGregor (1960) define la Teoría X como pragmática al indicar que solo se consideran los objetivos de la organización, el estilo de administrar es rígido y autocrático; con respecto al comportamiento humano se indica que las personas necesitan ser controladas, son perezosas y esquivan la responsabilidad. Por otro lado, la Teoría Y es participativa, en ella se desarrolla un estilo innovador, abierto, dinámico y participativo, crea oportunidades e impulsa el crecimiento. Las personas se esfuerzan, buscan responsabilidades se automotivan y autodirigen, son creativas y competentes (Villalta, 2000; López, 2007). 
La Teoría X presenta a los administradores una serie de razones fáciles de entender para explicar el funcionamiento ineficaz de la organización: se debe a la índole de los recursos humanos con los que hay que operar. En cambio, la Teoría Y dice que la solución a los problemas de la empresa es responsabilidad absoluta de la gerencia, que no conoce los recursos con los que cuenta. Si los empleados son perezosos, indiferentes, renuentes a aceptar responsabilidades, intolerantes, sin iniciativa ni espíritu de cooperación, la Teoría Y implica que las causas están en los métodos de organización y control de la gerencia (McGregor, p. 48).

El autor de esta teoría rescata además que el principio de integración exige que se reconozcan no solo las necesidades de la organización, sino también las del individuo.

\section{Teoría de la Motivación-Higiene (Frederick Herzberg)}

Esta teoría parte de que el hombre tiene un doble sistema de necesidades: la necesidad de evitar el dolor o las situaciones desagradables y la necesidad de crecer emocional e intelectualmente. Por ser cualitativamente distintos, cada tipo de necesidad, en el mundo del trabajo, requiere de incentivos diferentes. Por eso se puede hablar de dos tipos de factores que intervienen en la motivación en el trabajo:

1. Factores de higiene: procuran mantener un buen ambiente de trabajo, salarios justos, seguridad en el empleo, buenas relaciones con los funcionarios, entre otros.

2. Factores de motivación: ofrecen al funcionario su realización personal, obtener reconocimiento, aumentar el grado de responsabilidad y permitir que tengan más proba- bilidades de progreso dentro de la organización (Navajo, 2009).

Herzberg (1959) mencionaba respecto a la motivación que esta no se caracteriza como un rasgo personal, sino por la interacción de las personas y la situación, de ahí la importancia que los directivos dominen esto para que valoren y actúen, procurando que los objetivos individuales coincidan lo más posible con los de la organización (Cuadrado, 2010).

\section{Teoría de la Zanahoria y el Garrote}

Villalta (2000, p. 94) indica que esta teoría se basa en el uso de recompensas y castigos para influir en la conducta de la persona. Hace la relación entre el dinero como la "zanahoria" y "el garrote" a la acción de infundir temor acerca de perder el trabajo, destitución, reducción de bonos, entre otros.

No es que este sistema sea totalmente improductivo (sin duda las pirámides fueron construidas por los trabajadores que laboraban bajo promesa de una recompensa o la amenaza de la flagelación), pero ciertamente no es propicio para la creatividad y la innovación (Dowling, s.f.).

Esta teoría proviene básicamente de la narrativa que para lograr que se mueva un burro es necesario poner frente a él una zanahoria o golpearlo por detrás con un garrote. Por lo tanto, se podría considerar la zanahoria además, del salario ordinario, los incentivos, las promociones por antigüedad, bonos, aumentos salariales, que muchas veces se dan no por el desempeño del individuo sino por políticas internas o externas a la institución.

Además, el "garrote" ocasiona con frecuencia un comportamiento defensivo o represalias, como son la organización de sindicatos, el trabajo de baja calidad, la indiferencia en los ejecutivos e incluso la deshonestidad (Glzz, 2013). 


\section{Teoría de las necesidades secundarias (David McClelland)}

McClelland (1961) explica que una vez que el individuo logra satisfacer sus necesidades básicas (equivalentes a las mencionadas según la jerarquía de Maslow) la conducta de la persona requiere de otras tres necesidades. Estas son:

1. Necesidad de afiliación: deseo que tiene toda persona de mantener buenas relaciones con otros individuos y así disfrutar de su compañía. Poseen una elevada necesidad de afiliación y por lo general, son más sensibles a los sentimientos de otros, buscan establecer relaciones amistosas siendo agradables y apoyando ideas.

2. Necesidad de poder: el deseo de las personas es influir sobre otras, su característica es la lucha, la competencia y el prestigio.

3. Necesidad de logro: poseen un gran deseo de alcanzar el éxito, de plantearse constantemente metas desafiantes y realizar el esfuerzo necesario para llegar a cumplir lo que se propusieron (Stoner, 1994; Gross, 2009).

Cualquiera de estas teorías está sujeta al carácter del ser humano, lo que produce que su aplicación pueda ser todo un éxito o por el contrario llevar al fracaso.

Todas estas teorías tienen aspectos muy importantes a destacar y a utilizar hoy día, pues los seres humanos necesitan estar motivados para que su rendimiento laboral sea el más óptimo. Hay algunos factores que son necesarios para la vida de una persona, otros se van requiriendo con el pasar del tiempo, pero cualquiera de ellos es un parámetro a considerar para que los funcionarios se sientan realmente importantes en una organización creando empatía y con ello logren cumplir no solo sus objetivos personales sino también los laborales. También se puede decir que el castigo no es una buena razón a considerar, por el contrario se deben desarrollar buenas estrategias de compensación basadas en lo que la empresa pueda ofrecer.

A continuación se exponen ciertas consideraciones que se ajustan a algunas de estas teorías para generar motivación en el funcionario.

\section{Maneras de evitar la insatisfacción en el trabajo}

Hay ciertos puestos que provocan comportamientos rutinarios, los cuales así los considera el personal, afectando la satisfacción e identificación que el funcionario debe y puede tener con este. Para ello hay cinco maneras de evitar la rutina:

- $\quad$ Diversidad de trabajo: se refiere a la posibilidad de combinar varias funciones del trabajo, de manera que el personal tenga más operaciones por realizar, por ejemplo: dos o más trabajos se agregan en uno solo y hacen del trabajador una persona más calificada y proactiva con capacidad de desempeñar un trabajo sin estancarse en un solo puesto.

- La rotación en las actividades: con este sistema los funcionarios adquieren destrezas diferentes, les ofrece retos y les motiva al logro. En este caso como en el anterior se evita la monotonía y rutina en el trabajo.

- Enriquecimiento del trabajo: aumenta la profundidad de este, brinda la oportunidad para que cada cual establezca su propio ritmo de trabajo, corrige sus errores y escoge el mejor sistema para realizar las tareas, además permite intervenir en la toma de decisiones, aspecto que aumenta la responsabilidad y hace el trabajo más interesante.

- El horario: es un factor que influye en la satisfacción, pues horarios rígidos y cansados 
desmotivan al funcionario, por lo cual debe ser flexible y que permita cumplir con el trabajo. Aunque se han hecho muchos estudios y sugerencias sobre cuántas horas debe contemplar la jornada de trabajo, es más importante propiciar al trabajador condiciones óptimas para que se desempeñe con el máximo de satisfacción.

- El rediseño del puesto: consiste en implantar programas que ayuden a mejorar la situación actual del diseño del puesto cuando no es adecuado y afecta la productividad de la empresa, pues con el enriquecimiento se incrementa la motivación y mejora la exactitud y calidad del trabajo (OIT, 1984; Vásquez, 2011, p. 49).

Las teorías de motivación ayudan a los encargados de recursos humanos a definir qué sistemas de compensación son los más adecuados para el personal.

A partir de acá, se puede experimentar un cambio de paradigma en cuanto a los roles de trabajo y que a su vez le genere mayor motivación al funcionario; como lo promueve el teletrabajo.

\section{El teletrabajo y sus características}

Durante décadas, el trabajo se ha concebido como la realización de una actividad $u$ oficio a cambio de un salario, y que se ejecuta desde un lugar específico. En su gran mayoría ese lugar de trabajo es la oficina, o sedes de la empresa o institución para la que se labora. Sin embargo, desde hace más de cuarenta años, un científico de la Administración Nacional de la Aeronáutica y del Espacio de Estados Unidos (NASA) se dio a la tarea de investigar el cómo las tecnologías que existían en esa época, podían servir para algo más.

Nilles (1974) indicó que cuando comenzó a analizar el tema (él lo valoró desde su propia expe- riencia), la mayor eficiencia de tiempo y costos sobre el transporte que realizaba desde su lugar de residencia hasta su centro de trabajo. En ese momento Nilles concluyó que el teletrabajo no solo tenía futuro, sino que era una opción realmente válida como una modalidad laboral.

Durante años, Nilles lideró una investigación cuyo objetivo fue demostrar que al utilizar las tecnologías de información y comunicación, las personas podían laborar desde sus casas, sin necesidad de desplazarse hasta sus oficinas, con lo cual se reducían considerablemente los problemas de transporte y de polución. Además, concluyó que el teletrabajo mejoró la calidad de vida de los trabajadores y la calidad laboral en las empresas había aumentado.

Esta "nueva" modalidad laboral les permite a las instituciones y empresas de hoy ofrecerle a su recurso humano una opción para disminuir sus horas de traslado a cambio de entregas puntuales de sus proyectos, le facilita trabajar en horarios más flexibles, pues no se utiliza control de horas, más que la entrega de resultados y lo más importante, le permite al trabajador realizar sus actividades desde cualquier lugar.

Existen cuatro características fundamentales de la modalidad de teletrabajo:

1. Es realizada a distancia, por lo tanto no requiere de la presencia física del funcionario en la oficina.

2. Utiliza las diversas tecnologías de información y comunicación.

3. Existe una gran flexibilidad en el horario laboral del funcionario.

4. La rendición de cuentas es mediante la evaluación por resultados.

Hay múltiples expertos en esta temática. Tres de ellos son: Álvaro Lamé experto uruguayo y pro- 
motor del teletrabajo en su país; Francisco Ortiz, sociólogo, economista y autor de El teletrabajo: una nueva sociedad y Sonia Boiarov, actual Presidenta de la Academia Internacional de Teletrabajo y autora de múltiples investigaciones sobre teletrabajo en Latinoamérica.

Vivir acá, trabajar allá, ganar allá y gastar acá. El teletrabajo ofrece un alto potencial para que emprendedores y trabajadores del sector de servicios puedan aprovechar esta modalidad que transforma los espacios laborales, separando trabajo de oficina y cambiando la medición de horas por resultados (Lamé, 2014).

El criterio de Lamé recuerda inclusive que, ante la globalización, las personas están constantemente más expuestas a trabajar desde cualquier parte del mundo. Las grandes empresas transnacionales envían a diario a sus ejecutivos a otros países a realizar gestiones y a pesar de que estén fuera de sus centros de trabajo por una semana o varios días, su labor sigue realizándose exactamente igual a que si estuvieran presencialmente en su cubículo de trabajo.

El teletrabajo es propiciado por los avances en las tecnologías de la información y la comunicación, su nacimiento y proyección se basa en argumentos que le confieren una potencialidad de futuro susceptible de superar las distintas modas que, efectivamente, se introducen periódicamente en la vida empresarial y laboral. De acuerdo con estas premisas, el teletrabajo es un "producto" de la evolución y las tendencias en los campos de: la tecnología, la economía, la empresa, el empleo, el individuo y la sociedad (Ortiz, 2014).

Sin duda, las tecnologías evolucionan rápidamente. Los cambios que han sufrido las tecnologías de información y comunicación (TIC), la evolución, como dice Ortiz, han permitido que no solo el trabajo se pueda realizar a distancia, sino también las consultas médicas, los estudios, las compras, e inclusive hasta las relaciones interpersonales se logran vía Internet.

No obstante, y como se detallará más adelante, un requisito fundamental del teletrabajo es que medien las TIC, de otra manera no sería teletrabajo.

Nosotros realizamos diagnósticos en los que se propone la variante del teletrabajo para achicar costos y evitar despidos y reducciones salariales, intentando aportar desde la Carrera de Relaciones de Trabajo, una visión humanizante de las cuestiones laborales, que evite la variable de ajuste tradicionalmente aplicada en tiempos de crisis económica (Boiarov, 2014).

La selección del personal que va a teletrabajar es el principal pilar para que esta modalidad sea exitosa. Se debe evaluar a los teletrabajadores, buscar sus competencias básicas para cada tarea, elegir gente que tenga automotivación, disciplina, que sepa controlar sus tiempos, que pueda hacer su trabajo de forma independiente y que esté comprometida no solo con su tarea, sino con la empresa y con la experiencia de teletrabajo. Es conveniente realizar un estudio psico-laboral y ambiental antes de decidir la deslocalización (Boiarov, 2012).

La implementación del teletrabajo en una organización permite acciones estratégicas de modernización de la gestión, pues incorpora nuevos paradigmas en la gestión de trabajo.

Aunque hay riesgos y oportunidades que intervienen en la gestión de una fuerza de trabajo virtual, las oportunidades generalmente superan con creces los riesgos. Como explica Nilles, la clave para una fuerza de trabajo virtual exitosa es hacer el mejor uso de esas oportunidades a través de una adecuada planificación y el desarrollo de un estilo de gestión apropiada (Nilles, 1998). 
A continuación algunas características del teletrabajo, las cuales permiten, a la vez, vislumbrar un mejor contexto de cómo esta modalidad promueve cambios a la organización de una empresa.

\section{Es realizado a distancia}

El término "Teletrabajo" procede etimológicamente de la palabra griega tele que significa "lejos" y de trabajo, palabra latina que significa realizar una actividad física o intelectual. Por lo tanto, Teletrabajo significa realizar un trabajo desde lejos.

El trabajador decide el lugar en el que va a realizar sus actividades, en una gran mayoría opta por el domicilio; no obstante, también puede escoger alguna sede de la empresa o institución que esté más cerca de su residencia. Otras veces, decide teletrabajar desde otro lugar que le resulte más cómodo y óptimo para la ejecución de sus tareas, primando razones de tranquilidad, comodidad y pocas distracciones a su alrededor.

A su vez, se evitan traslados de muchos kilómetros y horas, lo que trae por consecuencia una reducción de estrés, una disminución en los gastos de combustibles, una reducción en la contaminación ambiental y por supuesto, estos cambios liberan un porcentaje significativo en la congestión vial.

Un estudio de la Cid-Gallup en agosto del 2013 en Costa Rica (La Nación, 2013), resaltó que los habitantes de la Gran Área Metropolitana pierden en promedio 15 días al año a causa de las presas.

El Club de Investigaciones Tecnológicas y Ernst \& Young llevaron a cabo una investigación en el 2013 en donde se obtuvieron los siguientes datos:

- En promedio una persona le toma desplazarse $25 \mathrm{~km}$ en ida y vuelta a su lugar de trabajo.
- El ahorro anual en caso de que una persona teletrabaje dos días a la semana aproximadamente sería de $\$ 627.500$ al año.

- Si una persona se traslada los cinco días a la semana a su oficina genera $2000 \mathrm{~kg}$ de CO2 al año, con lo cual se requerirían 50 árboles para mitigar dicha emisión de CO2. A diferencia de una persona que solo requiera trasladarse tres días a la semana, el CO2 que produce sería apenas de $800 \mathrm{~kg}$ de CO2 y por lo tanto requeriría de 20 árboles para mitigar dicha emisión.

Además, la ausencia de varios días a la semana del recurso humano, le permite a la empresa o institución realizar una optimización del espacio físico tanto en las oficinas como en las zonas de parqueo, trayendo múltiples beneficios para la institución, como por ejemplo: menos hacinamiento, mayor comodidad para quienes trabajan presencialmente, más espacios disponibles en el estacionamiento y, si es el caso, una reducción en el alquiler de edificios u oficinas.

\section{Utiliza las TIC}

Un requisito sine qua non del teletrabajo es que medien las tecnologías de información y comunicación (TIC), de otra manera no sería teletrabajo.

Comparar las tecnologías que existían en los años 70 con las que se cuentan en la actualidad, resultaría risible y desproporcional. Actualmente, la tecnología lo es todo. Existen teléfonos inteligentes desde los cuales se realizan videoconferencias o se interactúa inmediatamente con los contactos sin importar que estén en países diferentes; computadoras ultradelgadas y portátiles que permiten diseñar planos arquitectónicos o sistemas informáticos de grandes dimensiones, y hasta vehículos con sistemas de localización e Internet inalámbrico. 
Si Jack Nilles le vio futuro al teletrabajo hace cuatro décadas, sería imposible creer que las empresas e instituciones de hoy no puedan implementar el teletrabajo de forma exitosa, con las tecnologías que existen y que están en proceso.

Solo por mencionar algunas tecnologías básicas que contribuyen hoy en día a que una persona teletrabaje, desde su casa por ejemplo son: a nivel de hardware, computadoras con webcam y audífonos, teléfonos celulares inteligentes y por supuesto una conexión a Internet. A nivel de software, son múltiples las aplicaciones para comunicaciones integradas como chats, videoconferencias, Ilamadas por Internet, sistemas de almacenamiento de documentos desde la nube o bien por redes privadas, entre otros.

\section{Evaluación por resultados}

El teletrabajo aplicado en una organización, exige una planificación bajo la modalidad de trabajo por resultados, por metas o bien, por productos concretos. El sistema utilizado por muchos años atrás, en donde el trabajador solamente realizaba sus actividades en el marco de un horario, ya está obsoleto.

Una empresa o institución que desee implementar el teletrabajo, debe realizar un procedimiento claro de selección, pues no todos los puestos ni todas las personas pueden teletrabajar. En el caso que nos ocupa, no todas las actividades son teletrabajables. La rendición de cuentas debe realizarse con un instrumento que permita medir productos o metas, que defina fechas de entrega y exista un control de seguimiento de estos.

El jefe del teletrabajador no ejercerá una supervisión directa sobre su trabajo, ni sobre su presencia en la oficina, porque la persona estará laborando desde otro lugar. Por lo tanto, la evaluación del desempeño y de la gestión del teletrabajador deberá ser por resultados previamente planificados y programados.
Anteriormente, se indicaba que la estructura de una organización debe estar relacionada con las tareas, metas y objetivos. Es por esta razón, que el teletrabajo se vincula con una nueva estrategia organizacional.

Una correcta planificación del recurso humano y un mayor uso de las TIC influyen en mejores niveles de calidad en los servicios o productos que se generen, pero a la vez en una mayor productividad, pues hay una utilización más eficiente del tiempo, de los recursos humanos y de los materiales.

\section{Flexibilidad horaria}

Los horarios, en el teletrabajo, servirán para que a lo interno del departamento y de la lógica de la institución, logren las coincidencias que se requieran para reuniones, trabajos en grupo o cualquier otra necesidad de interacción sincrónica que se necesita entre varios compañeros de la empresa o institución.

Un teletrabajador podrá cambiar el horario que tenía, pues podría iniciar antes o después de la hora de inicio de aquellos que lo trabajen de forma presencial. Por razones obvias, el teletrabajador se economiza el tiempo de traslado a la oficina. Es así como una persona que dura aproximadamente una hora y media viajando de su casa a la oficina; podría iniciar las actividades desde su casa, una hora antes, por ejemplo.

En cuanto a esta característica de horarios flexibles, los jefes o patronos requieren tener un cambio de mentalidad pues, deben ejecutar su liderazgo basado en la confianza.

A la vez, la calidad de vida del teletrabajador, cambia significativamente al dedicarle más tiempo a sus actividades deportivas, culturales, sociales o familiares. El tiempo del teletrabajador se convierte en su completa responsabilidad, por lo cual se requieren personas responsables y con una alta capacidad de autorregulación. 
En la investigación mencionada anteriormente del Club de Investigaciones Tecnológicas y Ernst \& Young, el $86 \%$ de las personas encuestadas percibieron que el teletrabajo impactó de forma positiva su calidad de vida.

En el Libro Blanco del Teletrabajo se indica que "una de las condiciones básicas para implementar el teletrabajo como modelo organizacional radica en cómo se hace seguimiento y control a los resultados. Con esta modalidad quedan abolidos los seguimientos asociados al horario o la asistencia a un espacio puntual, por el contrario, se evalúa por resultados; esquema que impulsa la productividad y permite a cada empleado demostrar su potencial" (2010, p. 24).

\section{Vinculación del teletrabajo y las teorías de motivación}

Las autoras de esta investigación analizan la vinculación que puede tener el teletrabajo con las teorías de motivación antes expuestas.

En la teoría Jerarquía de Necesidades, se indica que el individuo tiene necesidades fisiológicas, de seguridad, de afecto o pertenencia, de estima y de autorrealización.

Según las características del teletrabajo permitiría al trabajador y según las necesidades fisiológicas, cierto descanso y mejor manejo de su tiempo, al permitirle generar sus actividades laborales en horarios flexibles; además, le proporciona al teletrabajador seguridad por medio de las políticas de personal pues el teletrabajo es una opción legal de trabajo, y parte de un principio fundamental que es la confianza en el trabajador, otro factor vinculado a la teoría como es la necesidad de estima.

Finalmente, el teletrabajo le permite a la persona utilizar todas sus potencialidades y habilidades, pues debe ser creativo y autorregularse, otra característica de esta teoría.
La Teoría del comportamiento X y Y, el teletrabajo contempla cada una de los conceptos de la teoría "Y" tanto desde la óptica de las organizaciones como para el individuo.

Según se indicó, la Teoría Y promueve que las personas sean participativas, que tengan un estilo innovador, abierto, dinámico y pretende que las personas se automotiven y autodirijan.

Trabajar a distancia implica que la persona debe tener ciertas competencias que le permitan ser un candidato idóneo para aplicar a esta modalidad. Según criterio técnico de la Oficina de Recursos Humanos de la Universidad Estatal a Distancia son siete competencias adicionales a las requeridas en un puesto, el cual debe tener una persona que opte por incorporarse a la modalidad de teletrabajo; estas son: automotivación, autorregulación, compromiso, comunicación, aprendizaje, orientación a la calidad y juicio. Muy consecuentes con lo que pretende la Teoría Y (Carvajal, 2012).

A su vez, un gerente orientado por la Teoría Y debe desarrollar un estilo de dirección democrático, participativo y permitiendo la autodirección y el autocontrol, lo que trae por consecuencia una autonomía a los trabajadores para que tomen decisiones sobre su trabajo. Escenario, sin duda, imprescindible para que el teletrabajo se dé de la mejor manera.

Además, y según McGregor, autor de esta teoría, indicaba que en la Teoría Y se aplica el principio de integración, el cual exige que se reconozcan no solo las necesidades de la organización, sino también las del individuo.

El teletrabajo promueve el reconocimiento de las necesidades que tiene no solo el individuo, sino la empresa y la sociedad también.

En cuanto a la Teoría de Motivación-Higiene, el teletrabajo tiene la cualidad de relacionarse directamente con los factores de motivación de 
esta teoría al ofrecer el logro, reconocimiento, trabajo desafiante y crecimiento laboral para los trabajadores.

Otro factor que promueve el teletrabajo y que coincide con esta teoría es que se preocupa por la vida personal del trabajador. Tal y como se indicó en un apartado anterior, el teletrabajo contribuye en la calidad de vida de la persona, pues este le permite destinar más tiempo para realizar actividades deportivas, académicas o familiares; se evitan el estrés generado por los traslados hasta sus oficinas y sin duda, genera un impacto económico a favor, pues disminuye los costos de traslados.

Boiarov incorpora un concepto interesante: el salario emocional. Según indica es la forma de compensación no económica que permite reconocer y atender necesidades de los empleados sin que ello signifique una mayor erogación para las empresas. Es una propuesta donde las dos partes se benefician (2012, p. 13).

La Teoría de la Zanahoria y el Garrote se basa en los conceptos de recompensas y castigos para influir en la conducta de una persona.

Casualmente el teletrabajo se podría considerar como una forma de recompensar al trabajador al darle la posibilidad de trabajar desde otro lugar, donde obtendría los beneficios que genera esta modalidad laboral.

A su vez, un ideal del teletrabajo es que se autorice incorporar a esta modalidad, a aquellos funcionarios que son responsables, cumplidos con sus tareas, organizados y que estén identificados con la Institución. Así, se les reconoce su buen trabajo y compromiso con que ha cumplido sus metas en el departamento.

El teletrabajo exige ciertas competencias especiales por parte de quien lo quiera ejecutar. Es por esto, que las jefaturas deben realizar de manera consensuada el proceso de selección del personal que desee aplicar a la modalidad de teletrabajo.

El teletrabajo podría definirse en la empresa o institución como un reconocimiento al funcionario que cuente con las competencias para un teletrabajador.

Un ejemplo que da fe de lo anterior es SunMicrosystems. Esta empresa tiene un programa de teletrabajo llamado "Open Work" y una encuesta reveló que ese programa es el motivo número uno por el que los empleados recomiendan trabajar ahí (Boiarov, 2012).

Y finalmente la Teoría de las necesidades secundarias explicaba sobre las necesidades de afiliación, de poder y de logro.

Un principio en materia de teletrabajo es que no todas las personas son aptas para teletrabajar, pues se requiere de ciertas competencias y además, se necesita que la persona esté en disposición de enfrentarse a un cambio en la forma en cómo ejecuta su trabajo. Por esa razón, el teletrabajo es voluntario.

Además, se puede destacar que la necesidad de afiliación y logro se ajustan perfectamente a los beneficios que da el teletrabajo. Por medio de esta modalidad se fomentan relaciones amistosas al acortar distancias con el uso de la tecnología y a su vez permite las metas y objetivos desafiantes. No cualquier persona, tiene el autocontrol para cumplir con su trabajo, en los plazos establecidos, cuando se está desde su casa, por ejemplo, a la vista de distracciones como son irse a dormir, a comer, o a pasear, pues "nadie lo está vigilando".

\section{Jack Nilles, las teorías de motivación y la productividad}

Desde la perspectiva del experto en teletrabajo, Jack Nilles, varios de los incentivos personales del teletrabajo se pueden reflejar en el modelo 
planteado por Maslow (Teoría de la Jerarquía de Necesidades).

Constantemente nos encontramos con que los teletrabajadores se sienten más al mando de sus vidas, son menos estresados, más creativos y más sanos que los compañeros que laboran desde la oficina. Sorprendentemente, también encontramos que los trabajadores a distancia parecen estar más en contacto con las actividades de los colegas en la oficina que los que están laborando de forma presencial. No sé si yo llamaría al teletrabajo en sí misma una teoría de la motivación, pero sí un medio para alcanzar los niveles más altos de la pirámide de Maslow (J. Nilles, comunicación personal, 12 de mayo del 2014).

Con respecto a las necesidades básicas de un trabajador, y cómo el teletrabajo podría contribuir a satisfacerlas, Nilles explica que las tres motivaciones que más a menudo hacen referencia los teletrabajadores son: evitar el estrés de los desplazamientos diarios hacia y desde el trabajo, el aumento del tiempo discrecional disponible y la reducción de las interrupciones en la ejecución del trabajo.

Además, indica que todos estos factores mejoran y contribuyen directamente en la productividad del trabajador y logran una mayor retención de los empleados o funcionarios antes de que estos se trasladen a otras empresas o instituciones.

Ciertamente, y según se ha venido detallando, el teletrabajo influye directamente con la productividad de un trabajador.

Llubere (2012) indica que la sostenibilidad de la práctica del teletrabajo se dará si se generan niveles superiores de desempeño en la ejecución de las actividades asignadas a los teletrabajadores que se traducen en resultados los cuales contribuyen con una mejora en la calidad y cantidad de los servicios brindados. También, si se refleja en acciones tangibles de valor para la organización y los clientes como reducción de costos, disminución de los tiempos de espera y menor efecto en el medio ambiente (2012, p. 35).

Además, explica que hay suficientes referencias para deducir lo que el cliente espera de las organizaciones, tales como: simplicidad, rapidez, autoservicio, trato personalizado, con poco o ningún papel y sin necesidad de pasar por escritorios obteniendo múltiples aprobaciones.

Con la tecnología disponible en la actualidad, prácticamente todas esas necesidades pueden ser satisfechas. Existen herramientas para hacer los flujos de trabajo muy eficientes, las páginas web brindan opciones de autoservicio cada vez mayores, la aplicación de la firma digital evita los desplazamientos y ahorra papel, el uso de las videocomunicaciones permite atender en tiempo real a clientes de lugares distantes, el teletrabajo móvil facilita ir donde el cliente y atender ahí sus requerimientos, también el teletrabajo fijo, ya sea en un telecentro o en el hogar, acorta los tiempos de respuesta (Llubere, 2012).

Desde esta perspectiva, si una persona que está teletrabajando logra la reducción en los tiempos de respuesta, o un aumento en la cantidad y calidad de sus productos finales, incrementa significativamente su productividad y por ende, la de su empresa o institución.

Jack Nilles afirma que son múltiples las empresas e instituciones que, una vez implementado el teletrabajo, han logrado demostrar un aumento en la productividad de sus trabajadores, como por ejemplo el estado de California, de Arizona y la Ciudad de Los Ángeles y las empresas IBM, AT\&T y la mayoría de las mejores empresas de Fortune 500, su productividad ha crecido de un $5 \%$ a un 30\%, dependiendo de la naturaleza del trabajo (J. Nilles, comunicación personal, 12 de mayo del 2014). 
En el Libro Blanco de Teletrabajo (2014) se cita el ejemplo de Cisco, en donde se refleja que la productividad ahora no se mide por el tiempo que pasa en las instalaciones de la oficina, sino por el avance medido y controlado sobre las metas propuestas para un periodo de tiempo establecido.

Según datos arrojados por Cisco, y en materia de productividad, se maneja una alentadora estadística de los resultados de sus clientes, quienes luego de implementar el teletrabajo obtuvieron incrementos en productividad que oscilan entre el 30\% y el 40\% (Libro Blanco, p. 36).

De igual manera, en la ciudad de Buenos Aires, Argentina, se realizó una investigación con el objetivo de analizar el impacto que el teletrabajo había generado tanto en los trabajadores como en la organización de diversas empresas.

Los datos arrojados en esta investigación, revelaron que las motivaciones para teletrabajar son como número uno la flexibilidad de horario con un $64 \%$, en segundo lugar, evitar traslados $62 \%$ y en tercer lugar el tiempo para la vida familiar, personal y laboral $61 \%$.

Entre las ventajas más importantes del teletrabajo indicaron mejora en la calidad de vida al poder contar con tiempo para compartir en familia con un $68 \%$ y tiempo para atender asuntos personales con un $62 \%$.

Además, las organizaciones adujeron que una ventaja para ellas fue haber mejorado la productividad.

Boiarov (2012) cita varios ejemplos concretos en donde se cuantifica mejor los beneficios obtenidos en las empresas que implementan esta modalidad:

1. IBM emplea al $25 \%$ de su personal por medio de teletrabajo (total de trabajadores: 320.000) y esto le ahorra 700 millones de dólares por operación y mobiliario.
2. AT\&T se beneficia con ahorros en costos de operación por 180 millones de dólares al año. La mayor parte de estos ahorros se debe a un aumento en la productividad y a la diminución de bienes inmuebles.

3. La calculadora de ahorros por teletrabajo del Telework Research Network de USA muestra que las compañías podrían ahorrarse cerca de 6500 dólares por cada teletrabajador que realice sus labores desde su casa una vez a la semana. (2012; p. 32)

\section{RESULTADOS Y CONCLUSIONES}

El objetivo general de esta investigación era demostrar que el teletrabajo podría considerarse una estrategia de motivación.

De acuerdo con el desarrollo de esta investigación, las autoras lograron:

1. Explicar qué es una teoría de motivación y resaltar las principales características de algunas de ellas.

2. Definir qué es el teletrabajo y cuáles son las principales ventajas y beneficios que les brinda a las empresas, a la sociedad y al trabajador.

3. Realizar una comparación sobre los beneficios que aporta el teletrabajo y las necesidades que requiere una persona en su vida, según las teorías de motivación.

4. Analizar los posibles aumentos en la productividad de las empresas que han implementado el teletrabajo.

La metodología empleada permitió analizar la documentación teórica sobre dos principales conceptos: teletrabajo y teorías de motivación; esto trajo por resultado una argumentación objetiva de la vinculación y articulación que puede existir entre ambos. 
Se consultaron estudios, investigaciones e informes relacionados con el tema del teletrabajo, la calidad de vida, la productividad y las motivaciones que requiere una persona. Y conjuntamente se incorporaron datos obtenidos de una entrevista realizada vía correo electrónico con el gurú del teletrabajo: Jack Nilles.

Todo esto, arroja como resultado grandes similitudes entre el teletrabajo y las teorías de motivación.

Se deduce de esta investigación que el teletrabajo es una estrategia de motivación que pueden aplicar las empresas.

Se concluye que la motivación es esencial para el desempeño individual de un trabajador y del comportamiento organizacional de una empresa o institución, lo que trae por consecuencia una mayor satisfacción y productividad para ambas partes.

Las teorías de motivación indican cuáles son las necesidades básicas de una persona y cómo son prioritarias estas en su vida.

El teletrabajo, con todas sus características, es una oportuna estrategia de motivación que aplican las organizaciones con el fin de obtener una mayor motivación por parte de sus funcionarios, pues les mejora su calidad de vida, se aumenta la productividad y se tiene más tiempo para las actividades personales.

El teletrabajo le transmite al funcionario, que su jefe y la institución confíen en él, que crean en su trabajo, que le permitan la ejecución de sus actividades desde otro lugar, fuera de las oficinas, sin un control presencial y que pueda administrar su tiempo.

Los teletrabajadores, sin duda, al tener cubiertas sus principales motivaciones generan una mayor productividad para la empresa, lo que trae por consecuencia beneficios significativos para su contratante.
Se podría concluir entonces que el teletrabajo aplica como una estrategia de motivación que permite al trabajador, realizar sus funciones desde cualquier lugar, en donde median las tecnologías de información y comunicación y que necesita de ciertos requisitos para que sea exitosa.

Se resume entonces de la siguiente manera:

\section{Teletrabajo, estrategia de motivación}

El teletrabajo motiva a que el funcionario opte de forma voluntaria a laborar desde su domicilio o cualquier otro lugar distinto al de su oficina. Para que se ejecute correctamente, quien opte por esta modalidad debe contar con siete competencias fundamentales: automotivación, autorregulación, compromiso, comunicación, aprendizaje, orientación a la calidad y juicio.

Tres necesidades que atiende el teletrabajo:

- Evita o disminuye el estrés ocasionado por los desplazamientos diarios hacia y desde la oficina.

- Aumenta el tiempo discrecional disponible.

- Reduce las interrupciones en la ejecución del trabajo.

Trae como resultado una mejor calidad de vida en el teletrabajador y un aumento en la productividad para la empresa.

La persona que teletrabaje potencia sus habilidades y capacidades, pues requiere ser innovador y responsable.

Promueve, a su vez, que el jefe de un teletrabajador practique un nuevo estilo de dirección, en donde su gestión mida resultados según objetivos y fechas planificadas y su supervisión sea indirecta. Y ante todo, demuestre confianza hacia sus trabajadores. 


\section{REFERENCIAS}

Barrantes, A. (22 de enero de 2014). Habitantes de GAM pierden 15 días al año en presas. La Nación. Recuperado de: http://www.nacion.com/nacional/HabitantesGAM-pierden-dias-presas_0_1392060835.html

Bolles, R. (1976). Teoría de la motivación, investigación experimental y evaluación. México: Editorial Trillas.

Boiarov, S. (2012). Lo que los líderes de Recursos Humanos deben saber sobre el teletrabajo. Cuadernos Profesionales. Argentina: Edición Fondo Editorial del CPCECABA.

Buere. G. (2003). Biografía y teoría de la personalidad de Abraham Maslow. Recuperado de: http://webspace.ship. edu/cgboer/maslowesp.html

Carvajal, L. (2012) Establecimiento de perfiles de personal que teletrabaja. San José: Oficina de Recursos Humanos, UNED.

Club de Investigaciones Tecnológicas. (2013). Investigación Teletrabajo: medio ambiente, economía y calidad de vida.

Cofer, C. y Appley, M. (1990). Psicología de la motivación, teoría e investigación. México: Editorial Trillas.

Corporación Colombia (2014). El Libro Blanco del Teletrabajo en Colombia. Recuperado de: http://www.colombiadigital.net/nuestras-publicaciones/empresarios/ item/2042-libro-blanco-el-abc-del-teletrabajo-encolombia.html

Cuadrado, N. (2010) Relación de los resultados de las pruebas de aprovechamiento en estudiantes de las escuelas elementales del distrito IV de la región educativa de San Juan y las funciones del director escolar como líder. Recuperado de: http://www.suagm.edu/umet/biblioteca/ UMTESIS/Tesis_Educacion/Adm_recreacion_deportes_2010/NCuadradoPastrana5152010.pdf

Dowling, E. (s.f). Definición de las teorías de motivación. Recuperado de: http://www.ehowenespanol.com/definicion-teorias-motivacion-sobre_98533/

Glzz, J. (2013). Teorías motivacionales. Recuperado de: http://clubensayos.com/Psicolog\%C3\%ADa/ Teor\%C3\%ADas-Motivacionales/1081636.html

Lamé A. (2014) Centro de Teletrabajo y Teleformación. Recuperado de: http://www.mintic.gov.co/portal/vivedigital/612/w3-article-4552.html)
López, C. (2007). Las teorías Xy Y de Douglas McGregor. Recuperado de: http://www.gestiopolis.com/canales/gerencial/articulos/18/xy.htm

Llubere, J. (ITA, 2012) La modernización de las organizaciones por medio del teletrabajo. Memoria Congreso Internacional Telework.

McGregor, D. (1994) El lado humano de las organizaciones. Bogotá, Colombia: McGraw Hill.

Las 8 teorías más importantes sobre la motivación. Nilles, J (1998). Managing Telework. Canadá: John Wiley \& Sons, Inc.

Centro de Teletrabajo y Teleformación.http://caminandoutopias.org.ar/contenidos/notas/rrhh/0014.php OIT. (1984) Serie Seguridad, higiene y medicina n. ${ }^{\circ}$ 56. Factores psicosociales en el trabajo, informe del Comité mixto OIT- OMS sobre medicina del trabajo. Ginebra. Recuperado de: http://www.google.com/url?sa=t\& $r c t=j \& q=\& e s r c=s \& f r m=1 \&$ source $=$ web $\& c d=24 \& v e d$ =0CGEQFjANOAo\&url=http\%3A\%2F\%2Fbiblioteca. uces.edu.ar\%2FMEDIA\%2FEDOCS\%2FFACTORES_ Texto.pdf\&ei = PfI_ VPnpLYvzgwT3 _ oHQDQ\&usg=AFQjCNGQqZZBIDG0e-TkuN9cVW38Cip ZJrQ\&bvm=bv.77648437,d.eXY

Stoner, J. (1994). Administración (5Ed.). México: Prentice Hall Hispanoamericana

Vásquez, N. (2011). Beneficios del Teletrabajo en la Universidad Estatal a Distancia. Costa Rica

Villalta, G. (2000). Curso Superior de Administración. Costa Rica: Editorial Sello Latino.

Recibido: 30 de agosto de 2014 Aceptado: 4 de octubre de 2014 